\title{
X-ray Imaging Using a Hybrid Photon Counting GaAs Pixel Detector
}

\author{
C. Schwarz ${ }^{a *}$, M. Campbell ${ }^{b}$, R. Goeppert ${ }^{a}$, E.H.M. Heijne ${ }^{b}$ J.Ludwig $^{a}$, G. Meddeler ${ }^{d}$, B. Mikulec ${ }^{b}$, \\ E. Pernigotti ${ }^{c}$, M.Rogalla ${ }^{a}$, K. Runge ${ }^{a}$, A. Söldner-Rembold ${ }^{a}$, K.M. Smith ${ }^{e}$, W. Snoeys ${ }^{b}$, J. Watt $^{e}$ \\ ${ }^{a}$ University of Freiburg, Hermann-Herder-Str. 3, 79104 Freiburg, Germany \\ ${ }^{b}$ CERN, Geneva, Switzerland \\ ${ }^{c}$ University and INFN of Pisa, Italy \\ ${ }^{d}$ Nikhef, Amsterdam, The Netherlands \\ ${ }^{e}$ University of Glasgow, Scotland
}

The performance of hybrid GaAs pixel detectors as X-ray imaging sensors were investigated at room temperature. These hybrids consist of $300 \mu \mathrm{m}$ thick GaAs pixel detectors, flip-chip bonded to a CMOS Single Photon Counting Chip (PCC). This chip consists of a matrix of $64 \times 64$ identical square pixels $(170 \mu \mathrm{m} \times 170 \mu \mathrm{m})$ and covers a total area of $1.2 \mathrm{~cm}^{2}$. The electronics in each cell comprises a preamplifier, a discriminator with a 3-bit threshold adjust and a 15-bit counter. The detector is realized by an array of Schottky diodes processed on semi-insulating LEC-GaAs bulk material. An IV-charcteristic and a detector bias voltage scan showed that the detector can be operated with voltages around $200 \mathrm{~V}$. Images of various objects were taken by using a standard $\mathrm{X}$-ray tube for dental diagnostics. The signal to noise ratio (SNR) was also determined.

The applications of these imaging systems range from medical applications like digital mammography or dental $\mathrm{X}$-ray diagnostics to non destructive material testing (NDT). Because of the separation of detector and readout chip, different materials can be investigated and compared.

\section{INTRODUCTION}

The most widely used detection medium for medical X-ray imaging is still photographic film. In the last few years, also digital X-ray imaging systems have been playing an increasing role. The main advantages of digital sensors in comparison to film systems are the higher sensitivity due to better absorption (this implies lower dose for the patient), the avoidance of time and material consuming chemical processing and the benefits of digital data handling like the possibility to apply software image processing tools to analyze the image.

The digital X-ray systems which are commercially available since a few years [1], mainly consist of silicon charge coupled devices (CCDs), with or without a scintillator conversion layer. Incident photons create electron hole pairs which are accumulated in potential wells formed by the electrodes of the CCD. These potential wells are

\footnotetext{
*Further author information: Tel: +49 761203 5935, Fax: +49 761203 5931, Email: Christoph.Schwarz@physik.unifreiburg.de
}

located very close to the surface of the CCD. In contrast to visible light which can be absorbed very well in this thin region, the absorption of $\mathrm{X}$-rays is much less efficient due to higher photon energy. To increase the absorption, the CCD is often covered with a scintillator layer. This concept has the disadvantage of decreasing image resolution and contrast because of scattering of conversion photons within the scintillator.

Another concept is given by hybrid pixel assemblies, which consist of a detector and a readout chip being connected together by a flip-chip process. Different developments have been sucessfully made especially for high energy physics and recently medical applications. A big advantage of the hybrid solution compared to monolithic devices like a CCD is the fact, that both chips can be optimized separately. While for the readout circuit the well known silicon CMOS technology is preferred, materials with an enhanced absorption efficiency for X-rays in the energy range of 10-70 keV such as GaAs or CdTe can be used.

A new step in the readout electronics is made 


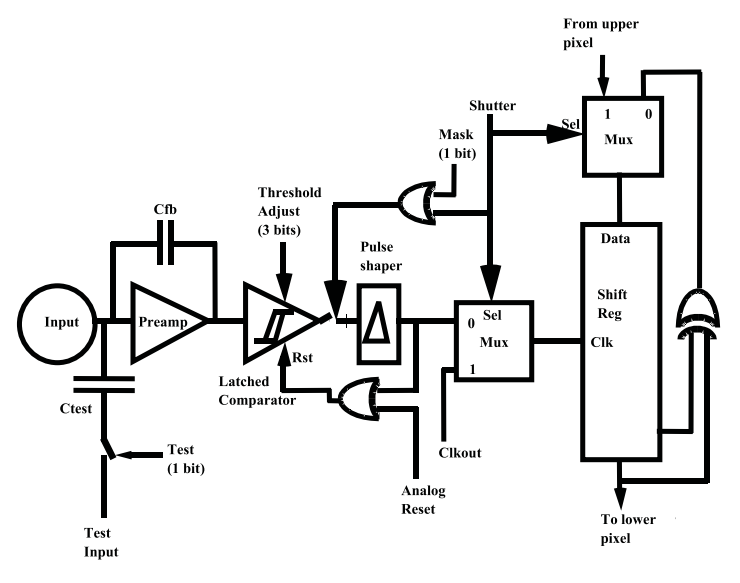

Figure 1. Block diagram of the pixel cell.

by using a single photon counting technique instead of an integrating method. This implies a faster read-out, low noise and a higher dynamic range. In this work, detectors processed on semiinsulating LEC-GaAs (SI-GaAs) bulk material and bump-bonded to the Photon Counting Chip (PCC) [2] were used.

\section{READOUT ELECTRONICS}

The PCC is a further development of the LHC1/Omega3 [3] chip, used in high energy physics, towards medical applications. It consists of a matrix of $64 \times 64$ matrix of identical square pixels $(170 \mu \mathrm{m} \times 170 \mu \mathrm{m})$ and covers a total sensitive area of $1.2 \mathrm{~cm}^{2}$. The electronics in each cell comprises a preamplifier with a leakage current compensation up to $10 \mathrm{nA} /$ pixel, an externally adjustable comparator with a 3 -bit fine tuning for each pixel, a short delay line which feeds back to the latched comparator to produce a pulse and a 15-bit pseudo-random counter. The input of the preamplifier is connected via a bump-bond to one of the detector pixels or can receive alternatively test signals from an external pulse generator via a test capacitance. When the shutter signal is low the pulse coming from the delay line becomes the clock of the counter. When the shutter is high, the feedback loop is broken and an external clock
Table 1

Summary of electrical measurements of the PCC [4].

\begin{tabular}{|c|c|}
\hline Minimum threshold & $1400 \mathrm{e}^{-}$ \\
\hline Threshold linearity & $\begin{array}{l}1400 \mathrm{e}^{-} \text {to } \\
7000 \mathrm{e}^{-}\end{array}$ \\
\hline $\begin{array}{l}\text { Threshold variation } \\
\text { (no adjust) }\end{array}$ & $350 \mathrm{e}^{-}$ \\
\hline $\begin{array}{l}\text { Threshold variation } \\
\text { (adjust) }\end{array}$ & $80 \mathrm{e}^{-}$ \\
\hline Noise & $170 \mathrm{e}^{-}$ \\
\hline Maximum input signal & $>80000 \mathrm{e}^{-}$ \\
\hline $\begin{array}{l}\text { Maximum counting } \\
\text { rate }\end{array}$ & $2 \mathrm{MHz}$ \\
\hline
\end{tabular}

can be used to shift out the data in a serial fashion. The maximum readout frequency is $10 \mathrm{MHz}$. There are two more fully static flip-flops to mask noisy pixel and to enable electrical testing.

A summary of electrical measurements of the PCC before bump-bonding is given in table 1 .

\section{DETECTOR - MATERIAL AND FABRICATION}

The detectors were fabricated in the Freiburg cleanroom facility on semi-insulating GaAs bulk material from FCM Freiberg, Germany. This material has typically a resistivity of $10^{7} \Omega \mathrm{cm}$. It has been shown that this type of GaAs has very good properties as a material for radiation detectors in high energy physics [5] and previously medical applications. The wafers were first lapped down from $650 \mu \mathrm{m}$ to $300 \mu \mathrm{m}$ and implanted on the backside with oxygen $\left(3 \cdot 10^{13} \mathrm{~cm}^{-2}\right.$ at $\left.190 \mathrm{keV}\right)$ to avoid backside firing. The Schottky contacts were processed on both sides by layers of $\mathrm{Ti}, \mathrm{Pl}$, $\mathrm{Au}$ and Ni. The front side is structured by photolithographic processes into a matrix of small pixels (gap: $10 \mu \mathrm{m}$ or $20 \mu \mathrm{m}$ ) of the same dimension as the readout electronics. The bond pads have a diameter of $20 \mu \mathrm{m}$, the passivation was made with a layer of $\mathrm{Si}_{3} \mathrm{~N}_{4}$. The so called underbump metallization for the flip-chip process was another layer of Au with an overlap of $2 \mu \mathrm{m}$. 


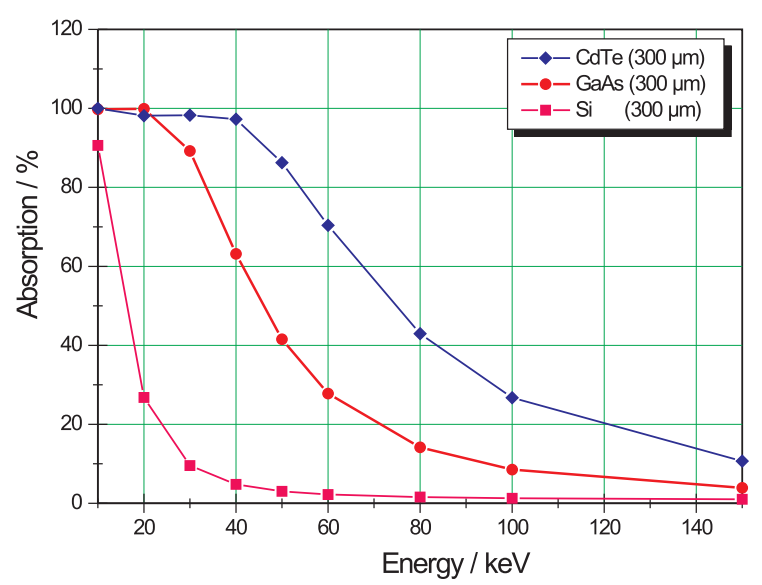

Figure 2. Absorption of X-rays in different materials.

In figure 2 the absorption probability of X-rays in the energy range 10 to $150 \mathrm{keV}$ for $\mathrm{Si}$, GaAs and CdTe, each $300 \mu \mathrm{m}$ thick, is plotted. It can be seen that the absorption of GaAs and CdTe in the interesting energy range 20 to $70 \mathrm{keV}$ is much higher than that of $\mathrm{Si}$ : for example at $30 \mathrm{keV}$, which is the peak of the X-ray spectra for $70 \mathrm{kV}$ tube voltage, the detection efficiency in $\mathrm{Si}$ is only $10 \%$, in contrast to GaAs with nearly $90 \%$. CdTe performs even better, but until now there are difficulties in terms of homogeneity and processing.

To determine the suitable reverse bias voltage settings of the detector, a IV-characteristic was taken with one assembly after the flip-chip bonding. A diode characteristic is expected. In figure 3 the leakage current in $\mu \mathrm{A}$ which flows into the detector is plotted as a function of the reverse bias voltage.

The characteristic has three distinct regions:

- a region where the leakage current increases linear with reverse bias voltage to reach a plateau,

- a saturation area in which the leakage current is approximately independent of the bias voltage,

- a region where the current increases again with the applied voltage (soft breakdown region).

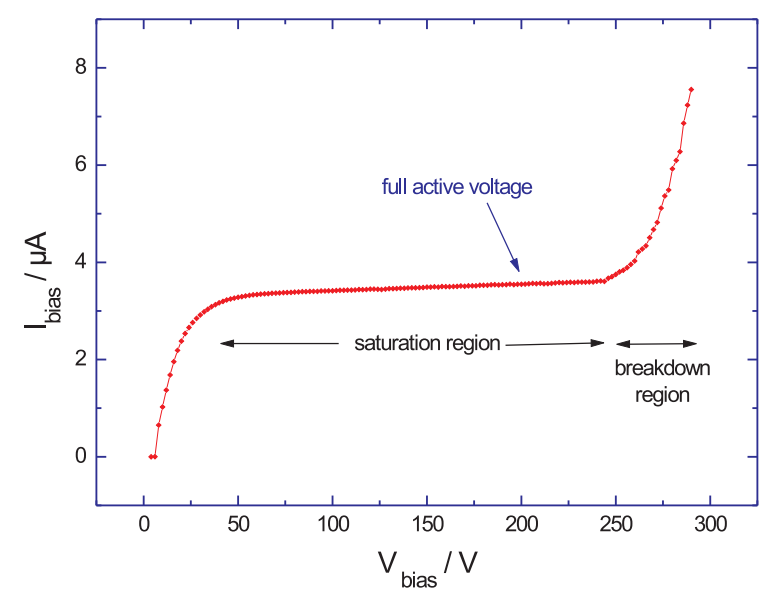

Figure 3. IV-characteristic of a flip-chip bonded assembly.

The soft breakdown is obtained due to the implantation of the backside of the wafer. The leakage current density measures $27 \mathrm{nA} / \mathrm{mm}^{2}$.

\section{IMAGING PROPERTIES}

To determine the imaging properties of the detector assembly we use a standard X-ray tube for dental diagnostics [?. Measurements using radioactive sources have been sucessfully done by other groups 河.

In a first measurement the assembly was exposed to a $200 \mathrm{~ms}$ long, $70 \mathrm{kV}$ X-ray pulse, and the mean counts per pixel for increasing reverse bias voltage were determined. We found that the mean counts per pixel reach a plateau at around $200 \mathrm{~V}$ (figure 1 ) and that there are almost no noisy pixels up to $250 \mathrm{~V}$ (figure 5). It should be mentioned that the bias settings of the readout chip were determined before, using an external pulse generator. The mean threshold of the pixels after adjustment was calculated to be $3794 \mathrm{e}^{-}$.

First images were taken from a $10 \mathrm{~mm}$ long M2 steel screw, placed on the back of the detector in a distance of $20 \mathrm{~cm}$ from the X-ray tube. The tube voltage was set to $60 \mathrm{kV}$, the exposure time

\footnotetext{
${ }^{1}$ Supplier: Siemens Type:Heliodent MD.
} 


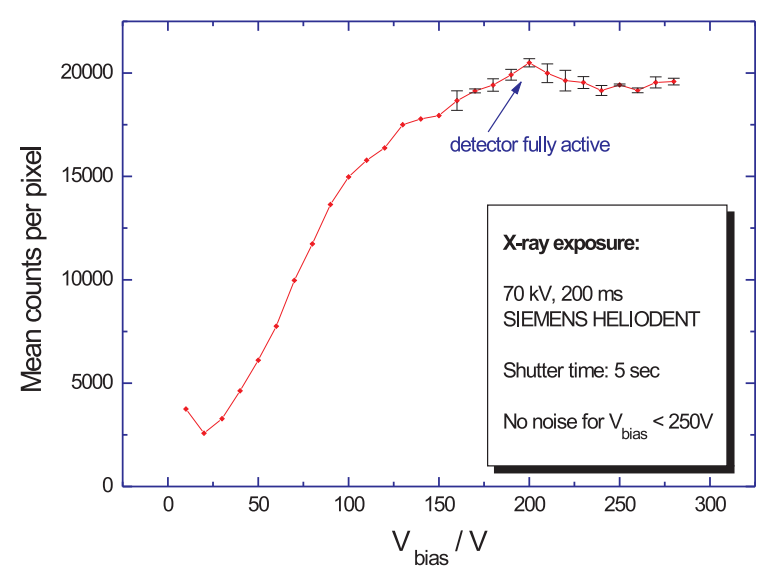

Figure 4. Bias voltage scan of a flip-chip bonded assembly: Mean counts per pixel.

to $50 \mathrm{~ms}$. These are nearly the minimum settings of the tube.

In figure 6 the raw data (the number of counts for each pixel) is plotted for the whole pixel matrix. The darker the pixel is plotted in the 8-bit greyscale, the higher the count rate and so the number of photons being detected in this pixel. Pixels which are plotted black, have counted more than 2500. All pixels are working, so the bumpbonding yield of this assembly seems to be nearly perfect. Nevertheless there are some small inhomogeneities, which can be attributed to variations in sensitivity of the detector.

This non-uniform sensitivity is probably a characteristic of the used bulk material. In semiinsulating LEC-GaAs, the deep donor arsenic antisite defect EL2 is normally used to compensate residual impurities with a flat energy level and is responsible for the semi-insulating behaviour. Otherwise the influence of flat acceptor concentrations like carbon would leave the material conducting.

It has been shown that these deep donors could limit the lifetime of charge carriers by acting as trapping centres [6]. Electron-hole pairs generated by incoming X-ray photons can be trapped on their way to the readout electrodes, so that only a fraction of the generated charge is detected. This implies a reduced charge collection

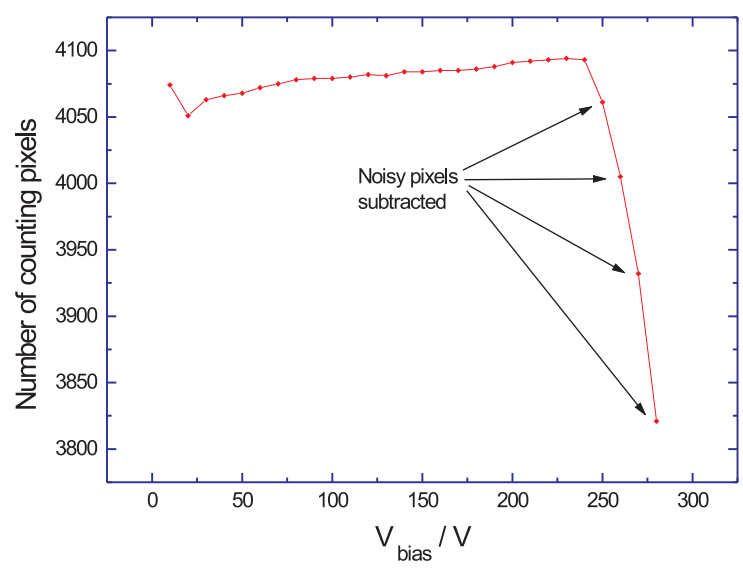

Figure 5. Bias voltage scan of a flip-chip bonded assembly: Number of counting pixels.

efficiency (CCE).

The local inhomogeneities also reduce the signal to noise ratio, which is defined as follows:

$\mathrm{SNR}=\frac{\text { signal }}{\text { noise }}=\frac{\langle\mathrm{n}\rangle}{\sigma}$

Here $\langle\mathrm{n}\rangle$ represents the mean number of counts per pixel in the region of interest and $\sigma$ is the standard deviation of the signal value. In case of photonic noise the SNR should have a square root dependency on the mean count rate as expected by the poisson statistic. Depending on the bias voltage of the detector, the exposure time to the X-rays and the optical density of the object and its spatial frequency, the SNR is not fixed. We obtained for the SNR a value of $4.1 \pm 0.1$ by taking a flood image, i.e. a uniform exposure of the whole detector, for $200 \mathrm{~V}$ bias voltage and a $100 \mathrm{~ms}$ long $\mathrm{X}$-ray pulse at $70 \mathrm{kV}$ tube voltage without applying any corrections to the data.

It has been shown [7] that in the case of time independent inhomogeneities in detector sensitivity an image correction method can be used to ameliorate the imaging properties. This method also increases the SNR by decreasing the $\sigma$. Further investigations will show if this method is also suitable for our detector system. Another possibility to get a better homogeneity is given by the threshold adjust facility of the PCC. Instead of 


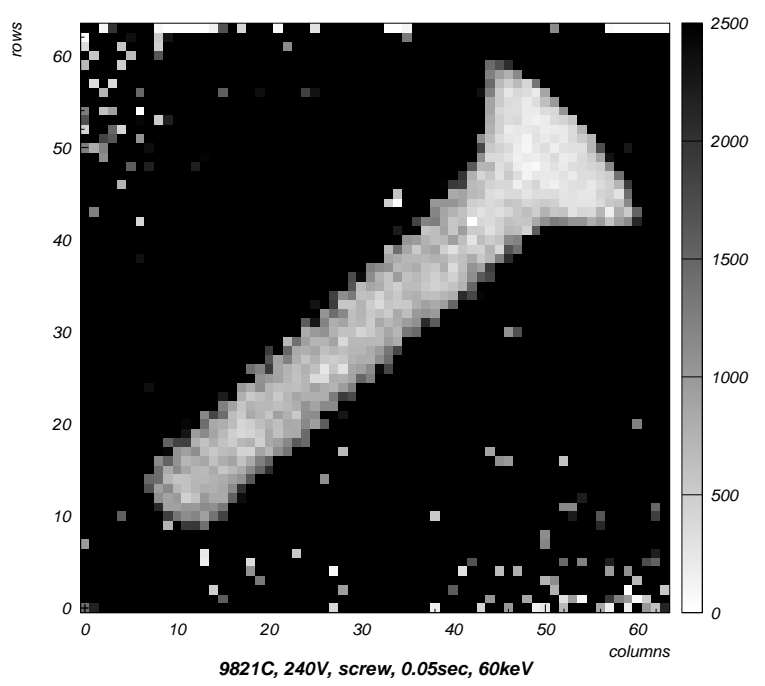

Figure 6. X-ray image of a M2 steel screw, $0.05 \mathrm{~s}$ at $60 \mathrm{kV}$.

adjusting the individual pixel threshold with a pulse on the test capacitance like it is done till now, an adjustment using the mean detector response to X-ray exposure could be carried out.

To improve the image quality in a first step, the image was inverted and interpolated. This is shown in figure 7. It should be mentioned that also the inner structure of the screw (thread, head) can be recognized.

There are many ways to evaluate the quality of an image. The most common and suitable methods are the contrast transfer function (CTF) and the modulation transfer function (MTF). The CTF describes the relative contrast response of an imaging system to a square wave modulation, the MTF the response to a sinusoidale one. They are both dependent on the spatial frequency whose unity is line pairs per $\mathrm{mm}(\mathrm{lp} / \mathrm{mm})$. The Nyquist frequency which is defined by $N_{y}=1 /(2 \times$ pitch $)$ measures $2.95 \mathrm{lp} / \mathrm{mm}$ for our detector system. Images of small slits down to the pixel size were sucessfully taken and the determination of the line spread function (LSF) and the corresponding MTF will be done soon.

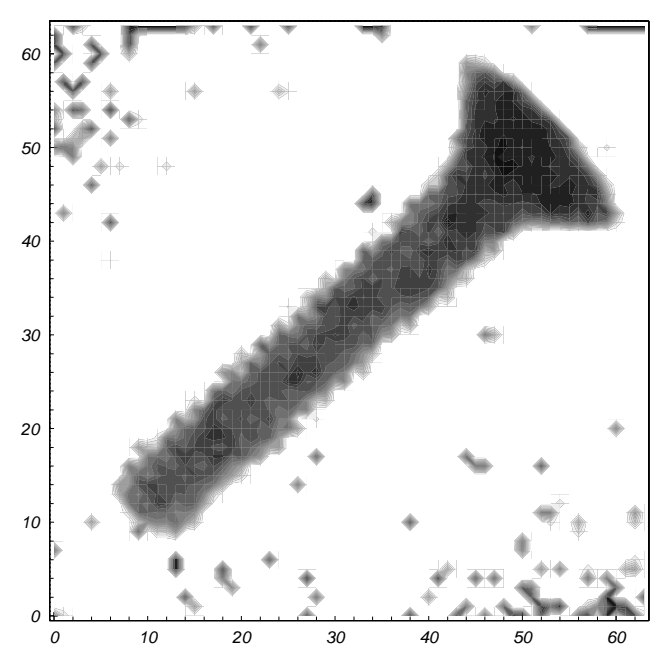

Figure 7. Interpolated and inverted X-ray image of a M2 screw, $0.05 \mathrm{~s}$ at $60 \mathrm{kV}$.

\section{CONCLUSION AND FUTURE WORK}

It has been shown that hybrid GaAs pixel detectors with photon counting electronics offer a promising alternative as digital X-ray imaging sensors. In this work SI-GaAs detectors, fabricated in Freiburg, were flip-chip bonded to 4096 Pixel Photon Counting Chips (PCC), developed at CERN. The leakage current density of the detector was determined by a IV-characteristic to $27 \mathrm{nA} / \mathrm{mm}^{2}$, which is in accordance to the expectation. A detector bias voltage scan showed that a voltage around $200 \mathrm{~V}$ is enough to have the detector fully active. There are almost no noisy pixel for voltages below $250 \mathrm{~V}$, the soft breakdown region of the detector.

Future work is given by investigations of the observed inhomogeneity in the taken X-ray images. If they can be attributed to variations in sensitivity of the detector and are time independent, an image correction methode can be developed and applied to the data. As a next step, characteristic quantities of an imaging system like the CTF and the MTF, will be determined and compared to other systems. 


\section{ACKNOWLEDGEMENTS}

This work was supported by the European Community under the Brite/Euram project XIMAGE (BE-1042). The readout chip was developed as part of the Medipix project, carried out by CERN, University of Freiburg, University of Glasgow and INFN-Pisa. We gratefully acknowledge the contribution of G. Humpston of GEC-Marconi Materials Technology Ltd., Caswell, England for the bumb-bonding, G. Magistrati of Laben S.p.A., Milano for the VMEbased readout system and M. Conti and collaborators of INFN-Napoli provided the readout software.

\section{REFERENCES}

1. U. Welander, "Resolution as defined by line spread and modulation transfer function for four digital intraoral radiographic systems", Oral Surg. Oral Med. Oral Pathol., vol 78, no.1, pp. 109-115, 1994

2. M. Campbell et al., "Readout for a $64 \times 64$ Matrix with 15-bit Single Photon Counting", IEEE Trans. Nucl. Sci. 45, pp. 751, 1998

3. E. H. M. Heijne et al., "LHC1: a semiconductor pixel detector readout chip with internal, tunable delay providing a binary pattern of selected events", Nucl. Instr. and Meth. A383, pp.52, 1996

4. M.G. Bisogni et. al., "Performance of a 4096 Pixel Photon Counting Chip" to be published in SPIE.

5. M. Rogalla et al., "Characterization of semiinsulating GaAs for detector applications", Nucl. Instr. and Meth. A380, pp. 14-17, 1996

6. M. Rogalla et al., "Analysis of trapping and detrapping in semi-insulating GaAs detectors", Nucl. Instr. and Meth. A395, pp. 49-54, 1997

7. R. Irsigler et al., "X-ray Imaging Using a $320 \times 240$ Hybrid GaAs Pixel Detector", to be published in IEEE. 